Pacific Journal of Mathematics

ORDER IDEALS IN CATEGORIES

auRelio Carbon and Ross Street 


\title{
ORDER IDEALS IN CATEGORIES
}

\author{
Aurelio Carboni and Ross Street
}

In the program to develop enriched category theory in a topos $\mathscr{E}$ it seems worthwhile to study the two particular bases $\Omega$ and $\mathbf{R}^{+}$; that is, the ordered objects of truth values and of non-negative extended reals with their appropriate monoidal structures. Categories in $\mathscr{E}$ enriched in $\Omega$ are ordered objects in $\mathscr{E}$, and it is this example we wish to study here.

Categories in $\mathscr{E}$ enriched in $\mathbf{R}^{+}$are metric spaces in $\mathscr{E}[\mathbf{8}]$ and the relevant $\mathbf{R}^{+}$has been studied in [10]. Since ordered objects occur at the very foundations of elementary topos theory, they have already been extensively studied (especially by Mikkelsen [9] and Brook [3]). However, our purpose is to emphasize the enriched-category viewpoint to give a guide to further development of the program.

Ordered objects can be defined without $\Omega$, of course, and much of the theory can be developed in a category $\mathscr{E}$ much more general than a topos. Our first two sections take this general approach. The first section deals with ideals in a regular category; from the enriched-category viewpoint these are the modules ( $=$ bimodules $=$ profunctors $=$ distributors). There is a bicategory $\operatorname{Idl}(\mathscr{E})$ of ordered objects and ideals. The first key result is that an ideal has a right adjoint if and only if it is locally principal. This means that locally principal ideals play the role that cauchy sequences play in metric space theory [8]. The question of whether every ordered object is "cauchy complete" thus becomes the question of whether locally principal implies principal. We show that this is true precisely when $\mathscr{E}$ satisfies the axiom of choice. The remainder of the first section deals with completeness of ordered objects.

The purpose of the second section is to construct, for ordered objects $A, B$, an object $[A, B]^{*}$ of order-preserving arrows from $A$ to $B$ with right adjoints and an object $[A, B]^{* *}$ of order-preserving arrows from $A$ to $B$ with right adjoints which have right adjoints. This requires $\mathscr{E}$ to be cartesian closed.

For the final section, $\mathscr{E}$ is required to be an elementary topos. For an ordered object $A$, we construct the object $\mathscr{P} A$ of order ideals in $A$ which, in enriched-category terms, is the object appropriate for receiving the yoneda embedding. After developing sufficiently the properties of $\mathscr{P} A$, we 
show that the cauchy completion of an ordered object $A$ is $2 A=$ $[\Omega, \mathscr{P} A]^{* *}$ (where the subobject classifier $\Omega$ is the value of $\mathscr{P}$ at the terminal object of $\mathscr{E}$ ).

For any bicategory $\mathscr{B}$, we write $\mathscr{B}^{*}$ for the sub-bicategory with the same objects and with the arrows which have right adjoints. We write $r^{*}$ for the right adjoint of a relation $r$ when it exists. Although we do consider right adjoints for order-preserving arrows and for ideals, we do not use the superscript ${ }^{*}$ for the right adjoints in these cases.

1. Order ideals. A relation $r: A \rightarrow B$ in a category $\mathscr{E}$ is a diagram $\left(r_{0}, R, r_{1}\right)$.

$$
A \underset{r_{0}}{\leftarrow} \underset{r_{1}}{\rightarrow} B
$$

such that, for all arrows $x, y: U \rightarrow R$, if $r_{0} x=r_{0} y$ and $r_{1} x=r_{1} y$ then $x=y$. An arrow $a: U \rightarrow A$ is r-related to an arrow $b: U \rightarrow B$ when there exists $x: U \rightarrow R$ with $r_{0} x=a, r_{1} x=b$; we write $a(r) b$.

An arrow $e: V \rightarrow U$ in $\mathscr{E}$ is called strong epic when, for all relations $r: A \rightarrow B$ and arrows $a: U \rightarrow A, b: U \rightarrow B$, if $a e(r) b e$ then $a(r) b$. A strong epic which is monic is invertible. Strong epic implies epic if $\mathscr{E}$ has pullbacks.

An ordered object $A$ of $\mathscr{E}$ consists of an object $A_{0}$ together with a relation $d=d_{A}=\left(d_{0}, A_{1}, d_{1}\right): A_{0} \rightarrow A_{0}$ such that, for all $a, b, c: U \rightarrow A_{0}$, the following conditions hold:

$$
\begin{gathered}
a\left(d_{A}\right) a \\
a\left(d_{A}\right) b, b\left(d_{A}\right) c \text { imply } a\left(d_{A}\right) c .
\end{gathered}
$$

An order-preserving arrow (or functor) $f: A \rightarrow B$ is an arrow $f: A_{0} \rightarrow B_{0}$ in $\mathscr{E}$ such that $a\left(d_{A}\right) a^{\prime}$ implies $f a\left(d_{B}\right) f a^{\prime}$. For order-preserving $f, f^{\prime}$ : $A \rightarrow B$, put $f \leq f^{\prime}$ when $f\left(d_{B}\right) f^{\prime}$. With the obvious composition, we obtain the bicategory $\operatorname{Ord}(\mathscr{E})$ of ordered objects in $\mathscr{E}$.

Objects of $\mathscr{E}$ are identified with ordered objects $A$ for which $d_{A}$ is the equality relation. When $\mathscr{E}$ has pullbacks, each arrow $h: V \rightarrow U$ in $\mathscr{E}$ gives an ordered object $E(h)=(V, d)$ where $x(d) y$ when $h x=h y$. Then $h$ : $E(h) \rightarrow U$ is order preserving.

For ordered objects $A, B$ in $\mathscr{E}$, an ideal $r: A \rightarrow B$ is a relation $r$ : $A_{0} \rightarrow B_{0}$ such that $a^{\prime}\left(d_{A}\right) a, a(r) b, b\left(d_{B}\right) b^{\prime}$ imply $a^{\prime}(r) b^{\prime}$.

In order to be able to compose relations and ideals usefully, we need conditions on the category. A category $\mathscr{E}$ is called regular when:

R1. pullbacks exist; 
R2. for all arrows $a: U \rightarrow A, b: U \rightarrow B$, there exists a relation $r=\left(r_{0}, R, r_{1}\right): A \rightarrow B$ and a strong epic $e: U \rightarrow R$ with $r_{0} e=a, r_{1} e=b$

R3. each pullback of each strong epic is strong epic.

For a regular category $\mathscr{E}$, there is a bicategory $\operatorname{Rel}(\mathscr{E})$ with the same objects as $\mathscr{E}$, with relations $r: A \rightarrow B$ as arrows, with a 2-cell $r \leq r^{\prime}$ if $r_{0}\left(r^{\prime}\right) r_{1}$, and composition of relations $r: A \rightarrow B, s: B \rightarrow C$ given by: $a(s r) c$ iff there exist $b$ and strong epic $e$ with $a e(r) b$ and $b(s) c e$.

Each $f: A \rightarrow B$ in $\mathscr{E}$ can be identified with $(1, A, f): A \rightarrow B$ in $\operatorname{Rel}(\mathscr{E})$. It is proved in [6] that an arrow $r$ in $\operatorname{Rel}(\mathscr{E})$ has a right adjoint $r$ * iff $r$ is isomorphic to an arrow in $\mathscr{E}$. The following result of André Joyal shows that our regular categories are regular in the sense of Barr [1].

Proposition 1. Each strong epic in a regular category is a coequalizer.

Proof. Let $p, q$ be the kernel pair of a strong epic $e$ (that is, the pullback of $e, e)$. Then $e e^{*}=1$ and $e^{*} e=q p^{*}$ in $\operatorname{Rel}(\mathscr{E})$. To show $e$ is the coequalizer of $p, q$, take $h$ with $h p=h q$. Put $r=h e^{*}$ in $\operatorname{Rel}(\mathscr{E})$. Then $r\left(e h^{*}\right)=h e^{*} e h=h q p^{*} h^{*}=(h p)(h p)^{*} \leq 1$ and $1=e e^{*} \leq e h^{*} h e^{*}$ $=\left(e h^{*}\right) r$. So $e h^{*}=r^{*}$ and $r \cong k$ where $k$ is in $\mathscr{E}$. Also $k e \leq r e=h e^{*} e$ $=h q p^{*}=h p p^{*} \leq h$ implies $k e=h$ since $k e, h$ are in $\mathscr{E}$. Since $e$ is epic, $k$ is unique with $k e=h$.

COROllary 2. An arrow $r$ in $\operatorname{Rel}(\mathscr{E})$ has a right adjoint iff there exists a strong epic e in $\mathscr{E}$ such that re is isomorphic to an arrow in $\mathscr{E}$.

Proof. If $r$ has a right adjoint then $e$ can be taken to be the identity. Conversely, if $r e \cong h$ with $h$ in $\mathscr{E}$ then $h p=h q$ for $p, q$ forming the kernel pair of $e$. By Proposition $1, h=g e$ for some $g$ in $\mathscr{E}$. So $r \cong r e e^{*} \cong$ $h e^{*} \cong g e e^{*} g$.

For a regular category $\mathscr{E}$, there is also a bicategory $\operatorname{Idl}(\mathscr{E})$ whose objects are the ordered objects in $\mathscr{E}$, whose arrows are ideals, and whose 2-cells and compositions are as for relations. The identity ideal of $A$ is $d_{A}$ : $A \rightarrow A$.

Each order-preserving arrow $f: A \rightarrow B$ yields an ideal $d_{B} f: A \rightarrow B$ which has a right adjoint $f^{*} d_{B}: B \rightarrow A$ in $\operatorname{Idl}(\mathscr{E})$. An ideal $r: A \rightarrow B$ is called principal when there exists an order-preserving arrow $f: A \rightarrow B$ such that $r \cong d_{B} f$. In general, not every ideal with a right adjoint is principal; however, Corollary 2 generalizes.

Proposition 3. An ideal $r: A \rightarrow B$ has a right adjoint iff there exists a strong epic e: $U \rightarrow A_{0}$ with $U$ in $\mathscr{E}$ and re: $U \rightarrow B$ principal. 
Proof. Suppose $r \multimap s$ in $\operatorname{Idl}(\mathscr{E})$. The unit condition $d_{A} \leq s r$ amounts to: $a\left(d_{A}\right) a^{\prime}$ implies there exist $b$ and strong epic $e$ with $a e(r) b$ and $b(s) a^{\prime} e$. The counit condition $r s \leq d_{B}$ amounts to: $b(s) a, a(r) b^{\prime}$ imply $b\left(d_{B}\right) b^{\prime}$. The unit condition with $a=a^{\prime}=1$ gives $e$ strong epic and $f$ with $e(r) f, f(s) e$. The counit condition using $f x(s) e x$, together with the ideal condition for $r$ using $\operatorname{ex}(r) f x$, yield that $\operatorname{ex}(r) b^{\prime}$ precisely when $f x\left(d_{B}\right) b^{\prime}$. Hence $x(r e) b^{\prime}$ precisely when $x\left(d_{B} f\right) b^{\prime}$. So $r e \cong d_{B} f$. Since the source of $f$ is in $\mathscr{E}$, order-preservingness is automatic. So re is principal.

Conversely, suppose $r e \cong d_{B} f$ with $e$ strong epic. Put $s=e f^{*}$. Then $r s=r e f^{*} \cong d_{B} f f^{*} \leq d_{B}$. So, to prove $r \rightarrow s$ it remains to prove $d_{A} \leq s r$. Suppose $a\left(d_{A}\right) a^{\prime}$ and let $x, e^{\prime}$ form a pullback for $e, a$. Now $x\left(d_{B} f\right) f x$ implies $x(r e) f x$ which implies $e x(r) f x$. Since $f x(s) e x, e x=a e^{\prime}$, $a e^{\prime}\left(d_{A}\right) a^{\prime} e^{\prime}$ and $s$ is an ideal, we have $f x(s) a^{\prime} e^{\prime}$. So we have $\operatorname{ex}(s r) a^{\prime} e^{\prime}$. So $a(s r) a^{\prime}$ because $e x=a e^{\prime}$ and $e^{\prime}$ is strong epic by R3.

Using the terminology of enriched category theory [3], we call an ideal cauchy when it has a right adjoint. An ordered object $X$ is cauchy complete when every cauchy ideal into it is principal; it follows from Proposition 3 that we only need to check for cauchy ideals with sources in $\mathscr{E}$. Thinking of strong epics as covers, we can interpret Proposition 3 as saying: an ideal is cauchy precisely when it is locally principal. We say that $\mathscr{E}$ satisfies the axiom of choice when every strong epic is a retraction.

COROLlary 4. The following three conditions on $\mathscr{E}$ are equivalent:

(i) the axiom of choice;

(ii) every ordered object is cauchy complete;

(iii) every equivalence is $\operatorname{Idl}(\mathscr{E})$ is principal.

Proof. (i) $\Rightarrow$ (ii) If $e$ is a retraction then re principal implies $r$ principal, so Proposition 3 gives the result.

(ii) $\Rightarrow$ (iii) Trivial.

(iii) $\Rightarrow$ (i) If $e: V \rightarrow U$ is a strong epic then $e: E(e) \rightarrow U$ is an equivalence in $\operatorname{Idl}(\mathscr{E})$. So $e^{*}: U \rightarrow E(e)$ is principal by (iii). Then $e^{*} \cong e^{*} e f$ with $f$ in $\mathscr{E}$; so $e f \cong e e^{*} e f \cong e e^{*} \cong 1$; so $e$ is a retraction.

The homomorphism

$$
\operatorname{Ord}(\mathscr{E})^{\mathrm{co}} \rightarrow \operatorname{Idl}(\mathscr{E})^{*}
$$

which is the identity on objects and takes $f$ to $d_{B} f$, is generally not a biequivalence (it is iff $\mathscr{E}$ satisfies the axiom of choice). Since $e: E(e) \rightarrow U$ 
is an equivalence in $\operatorname{Idl}(\mathscr{E})$ when $e$ is a strong epic, we obtain

$$
\operatorname{Ord}(\mathscr{E})(E(e), A)^{\mathrm{op}} \rightarrow \operatorname{Idl}(\mathscr{E})^{*}(E(e), A) \simeq \operatorname{Idl}(\mathscr{E})^{*}(U, A)
$$

taking $h: E(e) \rightarrow A$ to $d_{A} h e^{*}: U \rightarrow A$. Let CovU denote the ordered set whose elements are strong epics $e: V \rightarrow U$ (covers) with $e \leq e^{\prime}$ when there exists an arrow $f: V \rightarrow V^{\prime}$ such that $e=e^{\prime} f$. Notice that $(\mathrm{CovU})^{\text {op }}$ is a directed set by $\mathrm{R} 3$, and $e \mapsto E(e)$ gives a functor $E$ : $\operatorname{CovU} \rightarrow \operatorname{Ord}(\mathscr{E})$. Thus we have a cone of ordered sets:

$$
\operatorname{Ord}(\mathscr{E})(E-, A)^{\text {op }} \rightarrow \operatorname{Idl}(\mathscr{E})^{*}(U, A)
$$

Corollary 5. For $U$ in $\mathscr{E}$ and $A$ in $\operatorname{Ord}(\mathscr{E})$ the above cone induces an equivalence of ordered sets

$$
\underset{e \in \operatorname{Cov} U}{\operatorname{colim}} \operatorname{Ord}(\mathscr{E})(E(e), A)^{\mathrm{op}} \simeq \operatorname{Idl}(\mathscr{E})^{*}(U, A) .
$$

Proof. To obtain the inverse assignment, take a cauchy ideal $r$ : $U \rightarrow A$. Proposition 3 gives $r e \cong d_{A} h$ for some $h: V \rightarrow A$ and strong epic $e: V \rightarrow U$. Then $h e^{*} e \leq d_{A} h e^{*} e \cong r e e^{*} e \cong r e \cong d_{A} h$; so $h: E(e) \rightarrow A$ is order preserving.

For ideals $r: A \rightarrow C, s: B \rightarrow C$, we write $C(r, s): B \rightarrow A$ for the ideal characterized by the property:

$$
t \leq C(r, s) \text { iff } r t \leq s
$$

for all ideals $t: B \rightarrow A$. For a general $\mathscr{E}$, the ideal $C(r, s)$ may not exist for all $r, s$. If $r$ is cauchy then $C(r, s)$ is the composite of $s$ with the right adjoint for $r$; in particular, if $u$ : $A \rightarrow C$ is order preserving then $C\left(d_{C} u, s\right) \cong u^{*} s$.

Proposition 6. If $\mathscr{E}$ is finitely complete and each $\mathscr{E} / U$ is cartesian closed then $C(r, s)$ exists for all ideals $r: A \rightarrow C, s: B \rightarrow C$.

Proof. For ordered objects $A, B$, the inclusion of $\operatorname{Idl}(\mathscr{E})(A, B)$ in $\operatorname{Rel}(\mathscr{E})\left(A_{0}, B_{0}\right)$ whose value at a relation $r: A_{0} \rightarrow B_{0}$ is the relation (which happens to be an ideal) $A_{0} \rightarrow B_{0}$ obtained from the internal hom in $\mathscr{E} / A_{0} \times B_{0}$ of the objects $d_{1} \times d_{0}: A_{0} \times B_{0} \rightarrow A_{0} \times B_{0}$ and

$$
\left(\begin{array}{l}
r_{0} \\
r_{1}
\end{array}\right): R \rightarrow A_{0} \times B_{0}
$$


It is well known [7] that, under our conditions on $\mathscr{E}$, for each span $r$ : $U \rightarrow W$, the functor $\operatorname{Spn}(\mathscr{E})(V, U) \rightarrow \operatorname{Spn}(\mathscr{E})(V, W)$ obtained by composing with $r$ has a right adjoint. When $r$ is a relation this right adjoint induces a right adjoint to the functor $\operatorname{Rel}(\mathscr{E})(V, U) \rightarrow \operatorname{Rel}(\mathscr{E})(V, W)$ given by composing with $r$ in $\operatorname{Rel}(\mathscr{E})$.

For ideals $r: A \rightarrow C, s: B \rightarrow C$, the desired ideal $C(r, s)$ is the value of the right adjoint to

$$
\operatorname{Idl}(\mathscr{E})(B, A) \rightarrow \operatorname{Rel}(\mathscr{E})\left(B_{0}, A_{0}\right) \stackrel{r-}{\rightarrow} \operatorname{Rel}(\mathscr{E})\left(B_{0}, C_{0}\right)
$$

at $s$.

Suppose $r: A \rightarrow B$ is an ideal and $f: B \rightarrow X$ is order preserving. An $r$-weighted limit for $f$ is an order-preserving arrow $\lim (r, f): A \rightarrow X$ such that $\lim (r, f)^{*} d_{X} \cong B\left(r, f^{*} d_{X}\right)$.

Propositron 7. An ordered object $X$ is Cauchy complete iff $X$ admits all limits weighted by cauchy ideals.

Proof. For a cauchy ideal $r: A \rightarrow B$ with right adjoint $s$, we have $B\left(r, f^{*} d_{X}\right) \cong s f^{*} d_{X}$ which is a right adjoint for $d_{X} f r: A \rightarrow X$.

If $X$ is cauchy complete then $d_{X} f r \cong d_{X} g$ for some order preserving $g$; so $g^{*} d_{X} \cong B\left(r, f^{*} d_{X}\right)$ and $g \cong \lim (r, f)$.

If $X$ admits the indicated limits consider such $r$ with $B=X$. Let $g=\lim \left(r, 1_{X}\right)$ so that $g^{*} d_{X} \cong s 1_{X}^{*} d_{X} \cong s$. So $r \cong d_{X} g$ is principal.

Proposition 8. For any $j: A \rightarrow B$ in $\operatorname{Ord}(\mathscr{E})$, the functor

$$
\operatorname{Ord}(\mathscr{E})(j, 1): \operatorname{Ord}(\mathscr{E})(B, X) \rightarrow \operatorname{Ord}(\mathscr{E})(A, X)
$$

has right adjoint at $f: A \rightarrow X$ given by $\lim \left(j^{*} d_{B}, f\right)$ if this limit exists. If $j$ is fully faithful (i.e. $\left.d_{A} \cong j^{*} d_{B} j\right)$ then $\lim \left(j^{*} d_{B}, f\right) j \cong f$.

Proof. $g \leq \lim \left(j^{*} d, f\right)$ iff $g^{*} d \leq \lim \left(j^{*} d, f\right)^{*} d \cong B\left(j^{*} d, f^{*} d\right)$ iff $j^{*} d g^{*} d \leq f^{*} d$ iff $(g j)^{*} d \leq f^{*} d$ iff $g j \leq f$. If $j$ is fully faithful then $j^{*} d_{B} j f^{*} d_{X} \leq f^{*} d_{X}$, so $j f^{*} d_{X} \leq A\left(j^{*} d_{B}, f^{*} d_{X}\right) \cong \lim \left(j^{*} d_{B}, f\right)^{*} d_{X}$, so $f^{*} d_{X} \leq j^{*} \lim \left(j^{*} d_{B}, f\right) * d_{X}$, so $f \leq \lim \left(j^{*} d_{B}, f\right) j$.

These results relate our work to that of Bunge-Paré [5], Bunge [4] and Street [14].

2. Objects of adjunctions. Suppose the category $\mathscr{E}$ is finitely complete and cartesian closed:

$$
\mathscr{E}(U \times X, Y) \cong \mathscr{E}(U,[X, Y])
$$


For ordered objects $A, B$ in $\mathscr{E}$, form the pullback

$$
\begin{array}{ccc}
{[A, B]_{0}} & \rightarrow & {\left[A_{0}, B_{0}\right]} \\
\downarrow & & \downarrow\left(\begin{array}{l}
{\left[d_{0}, 1\right]} \\
{\left[d_{1}, 1\right]}
\end{array}\right) \\
{\left[A_{1}, B_{1}\right]} & \rightarrow \overrightarrow{\left.d_{0}\right]} & {\left[A_{1}, B_{0}\right] \times\left[A_{1}, B_{0}\right]}
\end{array}
$$

in which the horizontal arrows are monic. The order $d_{B}$ on $B_{0}$ induces an order on $\left[A_{0}, B_{0}\right]$ and hence on $[A, B]_{0}$ yielding an ordered object $[A, B]$ satisfying:

$$
\operatorname{Ord}(\mathscr{E})(C \times A, B) \cong \operatorname{Ord}(\mathscr{E})(C,[A, B]) .
$$

Indeed, $\operatorname{Ord}(\mathscr{E})$ is finitely complete and cartesian closed as a 2-category.

Proposition 9. For ordered objects $A, B$ in $\mathscr{E}$, there exists an ordered object $[A, B]^{*}$ with a natural equivalence of ordered sets:

$$
\mathscr{E}\left(U,[A, B]^{*}\right) \simeq \operatorname{Ord}(\mathscr{E} / U)^{*}(U \times A, U \times B)
$$

(where, of course, $U \times A, U \times B$ are regarded as objects of $\operatorname{Ord}(\mathscr{E} / U)$ by means of first projection onto $U$ ).

Proof. (This kind of result is folklore from the '60's; we indicate the proof for lack of a suitable reference.) The identity $[A, B] \rightarrow[A, B]$ corresponds to "evaluation" $\mathrm{ev}_{A}:[A, B] \times A \rightarrow B$, and the composite

$$
[B, C] \times[A, B] \times A \stackrel{1 \times \operatorname{ev}_{A}}{\rightarrow}[B, C] \times B \stackrel{\mathrm{ev}_{B}}{\rightarrow} C
$$

corresponds to "composition" $\operatorname{comp}_{B}:[B, C] \times[A, B] \rightarrow[A, C]$. The projection $1 \times A \rightarrow A$ gives $\operatorname{id}_{A}: 1 \rightarrow[A, A]$. Let $h: H \rightarrow[B, A] \times[A, B]$ denote the inserter (or subequalizer) of the pair of arrows

1

$$
[B, A] \times[A, B] \stackrel{\nearrow}{\underset{\operatorname{comp}_{B}}{\rightarrow}} \underbrace{\searrow i_{A}} \quad[A, A]
$$

in $\operatorname{Ord}(\mathscr{E})$; this means that an arrow $U \rightarrow H$ amounts to order-preserving arrows $f: U \times A \rightarrow B, g: U \times B \rightarrow A$ such that $p r_{3} \leq g(U \times f)$. Let $k$ : $K \rightarrow[A, B] \times[B, A]$ denote the inserter of the pair of arrows.

$$
\begin{aligned}
& {[A, B] \times[B, A] \stackrel{\operatorname{comp}_{A}}{\rightarrow}[B, B]} \\
& \searrow{ }_{1} \nearrow \mathrm{id}_{B}
\end{aligned}
$$


in $\operatorname{Ord}(\mathscr{E})$. Form the pullback

$$
\begin{array}{cccc}
{[A, B]_{0}^{*}} & & \rightarrow & K_{0} \\
\downarrow & & & \downarrow k \\
H_{0} & \rightarrow & {[B, A] \times[A, B] \cong[A, B] \times[B, A] .}
\end{array}
$$

It is easy to see that the composite of the above square with each projection onto $[A, B]$ and onto $[B, A]$ is monic. Let $[A, B]^{*}$ be the object $[A, B]_{0}^{*}$ enriched by the order induced from $[A, B]$ via the monic. The natural equivalence is easily verified.

There are order-preserving monics

$$
[A, B]^{*} \rightarrow[A, B] \text { and }[A, B]^{*} \rightarrow[B, A]^{\text {op }}
$$

induced by the inclusion

$$
\operatorname{Ord}(\mathscr{E} / U)^{*}(U \times A, U \times B) \rightarrow \operatorname{Ord}(\mathscr{E} / U)(U \times A, U \times B)
$$

and the right-adjoint-assigning monic

$$
\operatorname{Ord}(\mathscr{E} / U)^{*}(U \times A, U \times B) \rightarrow \operatorname{Ord}(\mathscr{E} / U)(U \times B, U \times A) .
$$

The object $[A, B]^{* *}$ defined by the pullback

$$
\begin{array}{ccc}
{[A, B]^{* *}} & \rightarrow & {[B, A]^{* \text { op }}} \\
\downarrow & & \downarrow \\
{[A, B]^{*}} & \rightarrow & {[B, A]^{\text {op }}}
\end{array}
$$

and the natural equivalence of ordered sets

$$
\mathscr{E}\left(U,[A, B]^{* *}\right) \simeq \operatorname{Ord}(\mathscr{E} / U)^{* *}(U \times A, U \times B)
$$

will be used to construct the cauchy completion of an ordered object.

Notice that these universal properties of $[A, B]^{*},[A, B]^{* *}$ do determine them up to isomorphism. This follows because $\mathscr{E} \rightarrow \operatorname{Ord}(\mathscr{E})$ is dense; in fact, $\mathscr{E} \rightarrow \operatorname{Cat}(\mathscr{E})$ is dense as can be seen using the extended Yoneda lemma [12; p. 287].

3. Cauchy completion. For this Section we assume that $\mathscr{E}$ is an elementary topos. Then $\mathscr{E}$ satisfies the assumptions of the earlier sections, including those of Proposition 6. The subobject classifier $\Omega$ is regarded as an ordered object via that order which gives a natural equivalence of ordered sets:

$$
\operatorname{Rel}(\mathscr{E})(X, Y) \simeq \mathscr{E}(X \times Y, \Omega)
$$


For ordered objects $A, B$, this equivalence (with $X=A_{0}, Y=B_{0}$ ) enriches to a natural equivalence

$$
\operatorname{Idl}(\mathscr{E})(A, B) \simeq \operatorname{Ord}(\mathscr{E})\left(A^{\text {op }} \times B, \Omega\right)
$$

where $A^{\text {op }}$ denotes $A_{0}$ with the reverse order $\left(d_{1}, A_{0}, d_{0}\right)$. Putting $A=$ $\left[\mathscr{P} A^{\mathrm{op}}, \Omega\right]$, we obtain a natural equivalence

$$
\operatorname{Idl}(\mathscr{E})(A, B) \simeq \operatorname{Ord}(\mathscr{E})(B, \mathscr{P} A)
$$

compare [11; pp. 172-5].

The identity of $\mathscr{P} A$ in $\operatorname{Ord}(\mathscr{E})$ corresponds to an ideal $\in_{A}: A \rightarrow \mathscr{P} A$ called membership. The last natural equivalence is then given by: the ideal $r: A \rightarrow B$ corresponds to the order-preserving arrow $f: B \rightarrow \mathscr{P} A$ when $r \cong f^{*} \in_{A}$; that is,

$$
a(r) b \text { iff } a\left(\in_{A}\right) f b .
$$

The yoneda embedding $y_{A}: A \rightarrow \mathscr{P} A$ is the order-preserving arrow defined by $d_{A} \cong y_{A}^{*} \in{ }_{A}$; that is,

$$
a \leq a^{\prime} \quad \text { iff } \quad a\left(\in_{A}\right) y_{A} a^{\prime} .
$$

Proposition 10. (i) $a\left(\in_{A}\right) f$ iff $y_{A} a \leq f$.

(ii) If $y_{A} a \leq f$ implies $y_{A} a \leq f^{\prime}$ for all a then $f \leq f^{\prime}$.

(iii) $\in_{A} y_{A}^{*} \cong d_{\mathscr{P} A}$.

(iv) The left extension of $y_{A}$ along $y_{A}$ is $1_{\mathscr{P} A}$ in $\operatorname{Ord}(\mathscr{E})$.

Proof. (i) $y_{A} a \leq f$ iff $a^{*} y_{A}^{*} \in f_{A} \leq f^{*} \in_{A}$ iff $a^{*} d_{A} \leq f^{*} \in \in_{A}$ iff $f a^{*} d_{A}$ $\leq \in_{A}$ iff $f a^{*} \leq \in_{A}$ (since $\in_{A}$ is an ideal) iff $a\left(\in_{A}\right) f$.

(ii) $f \leq f^{\prime}$ iff $f^{*} \in \in_{A} \leq f^{\prime *} \in \in_{A}$ iff $\left(a^{*} \leq f^{*} \in \in_{A} \Rightarrow a^{*} \leq f^{\prime *} \in_{A}\right)$ iff $\left(f a^{*} \leq \in_{A} \Rightarrow f^{\prime} a^{*} \leq \in_{A}\right)$ iff $\left(a\left(\in_{A}\right) f \Rightarrow a\left(\in_{A}\right) f^{\prime}\right)$ iff $\left(y_{A} a \leq f \Rightarrow\right.$ $\left.y_{A} a \leq f^{\prime}\right)$.

(iii) $p\left(\in_{A} y_{A}^{*}\right) q$ iff $\left(p e=y_{A} a, a\left(\in_{A}\right) q e\right.$ for some $a$ and epic $e$ ) iff ( $p e=y_{A} a, y_{A} a \leq q e$ for some $a$ and epic $e$ ) iff $p \leq q$ iff $p\left(d_{\mathscr{P}_{A}}\right) q$.

(iv) $y_{A} \leq k y_{B}$ iff $1\left(\in_{A}\right) k y_{A}$ iff $1\left(\in_{A} y_{A}^{*}\right) k$ iff $1\left(d_{\mathscr{P} A}\right) k$ iff $1 \leq k$.

An ordered object $X$ is called complete when it admits all limits weighted by all ideals.

Put $\mathscr{P}^{\dagger} A=\left(\mathscr{P} A^{\mathrm{op}}\right)^{\mathrm{op}}=[A, \Omega]^{\mathrm{op}}$ and $y_{A}^{\dagger}=\left(y_{A^{\mathrm{op}}}\right)^{\mathrm{op}}: A \rightarrow \mathscr{P}^{\dagger} A$. Then we have an ideal $\ni_{A}: \mathscr{P}^{\dagger} A \rightarrow A$ which induces an equivalence

$$
\operatorname{Ord}(\mathscr{E})\left(B, \mathscr{P}^{\dagger} A\right)^{\text {op }} \simeq \operatorname{Idl}(\mathscr{E})(B, A) \text {. }
$$

Proposition 11. For all $C \in \operatorname{Ord}(\mathscr{E})$ the ordered objects $\mathscr{P} C$ and $\mathscr{P}^{\dagger} C$ are both complete. 
Proof. Limits in $\mathscr{P}^{\dagger} C$ are obtained from composition of ideals. To see this take an ideal $r: A \rightarrow B$ and a functor $f: B \rightarrow \mathscr{P}^{\dagger} C$. Let $s: B \rightarrow C$ be the ideal corresponding to $f$ : this means $b(s) c$ iff $f b \leq y_{C}^{\dagger} c$. The composite ideal $s r: A \rightarrow C$ gives a functor $g: A \rightarrow \mathscr{P}^{\dagger} C$ with $g a \leq y_{C}^{\dagger} c$ iff $a(s r) c$. We claim that $g=\lim (r, f)$. Twice using Proposition 10(ii), we have $r t \leq f^{*} d$ iff $(p(t) a, a(r) b \Rightarrow p \leq f b)$ iff $\left(p(t) a, a(r) b, f b \leq y_{C}^{\dagger} c\right.$ $\left.\Rightarrow p \leq y_{C}^{\dagger} c\right)$ iff $\left(p(t) a, a(r) b, b(s) c \Rightarrow p \leq y_{C}^{\dagger} c\right)$ iff $(p(t) a, a(s r) c \Rightarrow p$ $\left.\leq y_{C}^{\dagger} c\right)$ iff $\left(p(t) a, g a \leq y_{C}^{\dagger} c \Rightarrow p \leq y_{C}^{\dagger} c\right)$ iff $(p(t) a \Rightarrow p \leq g a)$ iff $t \leq$ $g^{*} d$.

Limits in $\mathscr{P C}$ are obtained from right liftings of ideals (which exist by Proposition 6). To see this, take an ideal $r: A \rightarrow B$ and a functor $f$ : $B \rightarrow \mathscr{P} C$. Let $s: C \rightarrow B$ be the ideal corresponding to $f$. Let $g: A \rightarrow \mathscr{P} C$ be the functor corresponding to the ideal $B(r, s): C \rightarrow A$. One easily verifies that $g=\lim (r, f)$.

Proposition 12. The following conditions on an ordered object $X$ are equivalent:

(a) $X$ is complete;

(b) $y_{X}^{\dagger}: X \rightarrow \mathscr{P}^{\dagger} X$ has a right adjoint;

(c) $X^{\mathrm{op}}$ is complete;

(d) $y_{X}: X \rightarrow \mathscr{P} X$ has a left adjoint.

Proof. (a) $\Rightarrow$ (b) $\lim \left(\ni_{X}, 1_{X}\right): \mathscr{P}^{\dagger} X \rightarrow X$ can be verified to be a right adjoint for $y_{X}^{\dagger}$.

(b) $\Rightarrow$ (c) Condition (b) means that $y_{X^{\text {op }}}: X^{\text {op }} \rightarrow \mathscr{P}\left(X^{\text {op }}\right)$ has a left adjoint. Since $y_{X^{\text {op }}}$ is fully faithful and $\mathscr{P}\left(X^{\mathrm{op}}\right)$ admits all limits (Proposition 11), a familiar argument gives that $X^{\mathrm{op}}$ admits all limits and they are preserved by $y_{X^{\mathrm{op}} \text {. }}$

(c) $\Rightarrow$ (d) Apply (a) $\Rightarrow$ (b) to $X^{\text {op }}$.

(d) $\Rightarrow$ (a) Apply (b) $\Rightarrow$ (c) to $X^{\text {op }}$.

Proposition 13. If $X$ is complete then composition with $y_{A}: A \rightarrow \mathscr{P} A$ gives an equivalence

$$
\operatorname{Ord}(\mathscr{E})^{*}(\mathscr{P} A, X) \simeq \operatorname{Ord}(\mathscr{E})(A, X) .
$$

Furthermore, $[\mathscr{P} A, X]^{*} \simeq[A, X]$.

Proof. Composition with $y_{A}: A \rightarrow \mathscr{P} A$ gives a functor

$$
\operatorname{Ord}(\mathscr{E})(\mathscr{P} A, X) \rightarrow \operatorname{Ord}(\mathscr{E})(A, X)
$$

which has a left adjoint by Proposition 8 and 12; the left adjoint in fact lands in $\operatorname{Ord}(\mathscr{E})^{*}(\mathscr{P} A, X)$ since its value $\hat{f}: A \rightarrow X$ at $f: A \rightarrow X$ has a 
right adjoint $X \rightarrow \mathscr{P} A$ which corresponds to the ideal $d_{X} f: A \rightarrow X$. Since $y_{A}$ is fully faithful, this left adjoint $\operatorname{Ord}(\mathscr{E})(A, X) \rightarrow \operatorname{Ord}(\mathscr{E}) *(\mathscr{P} A, X)$ is fully faithful; since $y_{A}$ is dense (Proposition 10), it is surjective up to isomorphism. Thus we have the first equivalence. To obtain the second, apply the first in the topos $\mathscr{E} / U$ in place of $\mathscr{E}$, and use the denseness of $\mathscr{E} \rightarrow \operatorname{Ord}(\mathscr{E})$ with Proposition 9.

Using Proposition 10(iii), we see that we have a homomorphism of bicategories

$$
\operatorname{Idl}(\mathscr{E})^{\text {op }} \rightarrow \operatorname{Ord}(\mathscr{E})^{*}
$$

which is given on objects by $\mathscr{P}$ and on homs is the equivalence

$$
\operatorname{Idl}(\mathscr{E})(A, B) \simeq \operatorname{Ord}(\mathscr{E})(B, \mathscr{P} A) \simeq \operatorname{Ord}(\mathscr{E})^{*}(\mathscr{P} B, \mathscr{P} A)
$$

Since homomorphisms preserve adjunctions, we deduce that there is an equivalence

$$
\operatorname{Idl}(\mathscr{E})^{*}(A, B) \simeq \operatorname{Ord}(\mathscr{E})^{* *}(\mathscr{P} A, \mathscr{P} B)
$$

Apply this now to the ordered objects $U$ and $U \times A$ in the topos $\mathscr{E} / U$ to obtain:

$$
\begin{aligned}
\operatorname{Idl}(\mathscr{E})^{*}(U, A) & \simeq \operatorname{Idl}(\mathscr{E} / U)^{*}(U, U \times A) \\
& \simeq \operatorname{Ord}(\mathscr{E} / U)^{* *}(U \times \Omega, U \times \mathscr{P} A)^{\mathrm{op}} \\
& \simeq \mathscr{E}\left(U,[\Omega, \mathscr{P} A]^{* *}\right)^{\mathrm{op}}
\end{aligned}
$$

THEOREM 14. Each ordered object $A$ in an elementary topos $\mathscr{E}$ has a cauchy completion $2 A$. In fact, $\mathscr{2} A=[\Omega, \mathscr{P} A]^{* *}$ is cauchy complete and there exists a fully faithful functor $n_{A}: A \rightarrow \mathscr{Q} A$ which, for all cauchy complete $X$, induces an equivalence of ordered sets

$$
\operatorname{Ord}(\mathscr{E})(\mathscr{Q} A, X) \simeq \operatorname{Ord}(\mathscr{E})(A, X)
$$

Proof. The following equivalence is proved above:

$$
\mathscr{E}(U, \mathscr{Q} A) \simeq \operatorname{Idl}(\mathscr{E})^{*}(U, A)^{\text {op }} .
$$

The natural functors

$$
\mathscr{E}(U, A) \rightarrow \operatorname{Idl}(\mathscr{E})^{*}(U, A)^{\mathrm{op}} \rightarrow \operatorname{Idl}(\mathscr{E})(A, U)
$$


(the first takes $f$ to $d_{A} f$ and the second takes a cauchy ideal to its right adjoint) induce fully faithful functors

$$
A \underset{n_{A}}{\rightarrow} \mathscr{Q} A \underset{m_{A}}{\rightarrow} \mathscr{P} A
$$

between the representing objects such that $m_{A} n_{A} \cong y_{A}$. The equivalence (a) therefore takes $f: U \rightarrow \mathscr{Q} A$ to the left adjoint of the ideal $f^{*} m_{A} \in_{A}$. There will be no ambiguity in omitting the subscripts from $m_{A}, n_{A}$, and so on.

We shall show that the fully faithful functor

$$
\operatorname{Idl}(\mathscr{E})^{*}(U, A) \rightarrow \operatorname{Idl}(\mathscr{E})^{*}(U, \mathscr{Q} A)
$$

which takes $r$ to $d n r$, is an equivalence. To see this, take a cauchy ideal $s$ : $U \rightarrow \mathscr{2} A$. By Proposition 3, there exist a (strong) epic $e: V \rightarrow U$ and arrow $f: V \rightarrow \mathscr{2} A$ with $s e \cong d f$. Under (a) the arrow gives a cauchy ideal $t: V \rightarrow A$ whose right adjoint ideal is $f^{*} m^{*} \in$. So we obtain a cauchy ideal $r \cong t e^{*}: U \rightarrow A$. Now $f^{*} m^{*} \in n^{*} d \cong f^{*} m^{*} \in n^{*} m^{*} m d \cong f^{*} m^{*} \in$ $y^{*} m d \cong f^{*} m^{*} d m d \cong f^{*} d$, so, taking left adjoint ideals, we obtain $d n t \cong$ $d f \cong s e$; so $s \cong d n t e^{*} \cong d n r$. So $s$ is, up to isomorphism, in the image of (b).

Combining (a), (b), we obtain the equivalence

$$
\mathscr{E}(U, \mathscr{Q} A)^{\text {op }} \simeq \operatorname{Idl}(\mathscr{E})^{*}(U, \mathscr{Q} A) .
$$

Thus $\mathscr{Q} A$ is cauchy complete.

Next we show that the ideal $n^{*} d: \mathscr{2} A \rightarrow A$ is cauchy. Composition with $d n$ is a fully faithful functor

$$
\operatorname{Idl}(\mathscr{E})(U, A) \rightarrow \operatorname{Idl}(\mathscr{E})(U, \mathscr{Q} A)
$$

whose right adjoint is composition with $n * d$. Furthermore, this adjunction restricts to the equivalence (b). It follows that composition with $n^{*} d$ gives the inverse equivalence for (b). Thus $n^{*} d f$ is cauchy for all functors $f$ : $U \rightarrow \mathscr{Q} A$. By Proposition $3, n^{*} d$ is cauchy.

If $X$ is cauchy complete then it admits limits weighted by $n * d$ (Proposition 7). The functor

$$
\operatorname{Ord}(\mathscr{E})(\mathscr{Q} A, X) \rightarrow \operatorname{Ord}(\mathscr{E})(A, X)
$$

given by composition with $n$ thus has a right adjoint (Proposition 8) which is fully faithful since $n$ is. It remains to show that (d) reflects isomorphisms. Since every $X$ has a fully faithful functor $y_{X}: X \rightarrow \mathscr{P} X$ into a complete object, it suffices to prove (d) reflects isomorphisms for $X$ complete. 
Let $m^{\dagger}: A \rightarrow \mathscr{P}^{\dagger} A$ denote the fully faithful functor induced by the inclusion

$$
\operatorname{Idl}(\mathscr{E})^{*}(U, A)^{\mathrm{op}} \rightarrow \operatorname{Idl}(\mathscr{E})(U, A)^{\mathrm{op}}
$$

then $y^{\dagger}: A \rightarrow \mathscr{P}^{\dagger} A$ is isomorphic to $m^{\dagger} n$. Suppose $g, h: \mathscr{Q} A \rightarrow X$ are functors with $g \leq h$ and $g n \cong k n$. Assuming $X$ complete, we have right extensions $f^{\prime}, h^{\prime}: \mathscr{P}^{\dagger} A \rightarrow X$ of $g, h$ along $m^{\dagger}$ in $\operatorname{Ord}(\mathscr{E})$ with $g^{\prime} m^{\dagger} \cong g$, $h^{\prime} m^{\dagger} \cong h$. So $g^{\prime} y^{\dagger} \cong g^{\prime} m^{\dagger} n \cong g n \cong k n \cong h^{\prime} m^{\dagger} n \cong h^{\prime} y^{\dagger}$. By the dual of Proposition 13, we have $g^{\prime} \cong h^{\prime}$. Hence $g \cong g^{\prime} m^{\dagger} \cong h^{\prime} m^{\dagger} \cong k$.

Corollary 15. (a) $\mathscr{P} \mathscr{Q} A \simeq \mathscr{P} A$. (b) $\mathscr{P} A \simeq \mathscr{P} B$ iff $\mathscr{Q} A \simeq \mathscr{Q} B$.

Proof. (a) Since $\mathscr{P}^{\dagger} B$ is cauchy complete, we have

$$
\begin{aligned}
\operatorname{Ord}(\mathscr{E})(B, \mathscr{P} \mathscr{Q} A) & \simeq \operatorname{Idl}(\mathscr{E})(\mathscr{Q} A, B) \\
& \simeq \operatorname{Ord}(\mathscr{E})\left(\mathscr{Q} A, \mathscr{Q}^{\dagger} B\right)^{\mathrm{op}} \simeq \operatorname{Ord}(\mathscr{E})\left(A, \mathscr{P}^{\dagger} B\right)^{\mathrm{op}} \\
& \simeq \operatorname{Idl}(\mathscr{E})(A, B) \simeq \operatorname{Ord}(\mathscr{E})(B, \mathscr{P} A) .
\end{aligned}
$$

So (a) follows.

(b) From the formula $\mathscr{Q}-=[\Omega, \mathscr{P}-]^{* *}$ we see that $\mathscr{P} A \simeq \mathscr{P} B$ implies $\mathscr{Q} A \simeq \mathscr{Q} B$. The converse follows from (a).

\section{REFERENCES}

[1] M. Barr, Exact Categories, Lecture Notes in Math., 236 (Springer, Berlin-New York, 1971), 1-120.

[2] R. Betti, A. Carboni, R. Street and R. Walters, Variation through enrichment, J. Pure and Appl. Algebra, 29 (1983), 109-127.

[3] T. Brook, Order and Recursion in Topoi, Notes on Pure Mathematics, 9 (Australian National University, Canberra, 1977), 226 pp.

[4] M. Bunge, Stack completions and Morita equivalence for categories in a topos, Cahiers de topologie et géometrie différentielle, 20 (1979), 401-435.

[5] M. Bunge and R. Paré, Stacks and equivalence of indexed categories, Cahiers de topologie et géometrie différentielle, 20 (1979), 373-399.

[6] A. Carboni, S. Kasangian and R. Street, Bicategories of spans and relations, J. Pure and Appl. Algebra, 33 (1984), 259-267.

[7] B. J. Day, Limit Spaces and Closed Span Categories, Lecture Notes in Math., 420 (Springer, Berlin-New York, 1974), 65-74.

[8] F. W. Lawvere, Metric spaces, generalized logic, and closed categories, Rend. Sem. Mat. Fis. Milano, 43 (1973), 135-166.

[9] C. J. Mikkelsen, Lattice-theoretic and logical aspects of elementary topoi, Aarhus Universitet Various Publication Series, 25 (1976).

[10] J. Z. Reichman, Semicontinuous real numbers in a topos, J. Pure and Appl. Algebra, 28 (1983), 81-91. 
[11] R. Street, Elementary Cosmoi, Lecture Notes in Math., 420 (Springer, Berlin-New York, 1974), 134-180.

[12] _ Cosmoi of internal categories, Trans. Amer. Math. Soc., 258 (1980), 271-318.

[13] _ Cauchy characterization of enriched categories, Rend. Sem. Mat. Fis. Milano, 51 (1981), 217-233.

[14] _ Characterization of Bicategories of Stacks, Lecture Notes in Math., 962 (Springer, Berlin-New York, 1982), 282-291.

[15] __ Enriched categories and cohomology, Quaestiones Math., 6 (1983), 265-283.

Received February 15, 1984.

ISTItUto MATEMatico “Federigo EnRiQues”

VIA SALDINI 50

20122 Milano, ITALY

AND

MACQUARIE UNIVERSITY

NORTH RYDE, 2113 AUSTRALIA 


\section{PACIFIC JOURNAL OF MATHEMATICS EDITORS}

\author{
V. S. VARADARAJAN \\ (Managing Editor) \\ University of California \\ Los Angeles, CA 90024 \\ Herbert Clemens \\ University of Utah \\ Salt Lake City, UT 84112 \\ R. FINN \\ Stanford University \\ Stanford, CA 94305
}

\author{
HERMANN FLASCHKA \\ University of Arizona \\ Tucson, AZ 85721 \\ RAMESH A. GANGOLLI \\ University of Washington \\ Seattle, WA 98195 \\ VAUGHAN F. R. JONES \\ University of California \\ Berkeley, CA 94720 \\ ROBION KIRBY \\ University of California \\ Berkeley, CA 94720
}

C. C. MOORE

University of California

Berkeley, CA 94720

H. SAMELSON

Stanford University

Stanford, CA 94305

HAROLD STARK

University of California, San Diego La Jolla, CA 92093

\section{ASSOCIATE EDITORS}
R. ARENS
E. F. BECKENBACH (1906-1982)
B. H. NEUMANN
F. WOLF
K. YosHIDA

\section{SUPPORTING INSTITUTIONS}

\begin{abstract}
UNIVERSITY OF ARIZONA
UNIVERSITY OF BRITISH COLUMBIA

CALIFORNIA INSTITUTE OF TECHNOLOGY

UNIVERSITY OF CALIFORNIA

MONTANA STATE UNIVERSITY

UNIVERSITY OF NEVADA, RENO

NEW MEXICO STATE UNIVERSITY OREGON STATE UNIVERSITY
\end{abstract}

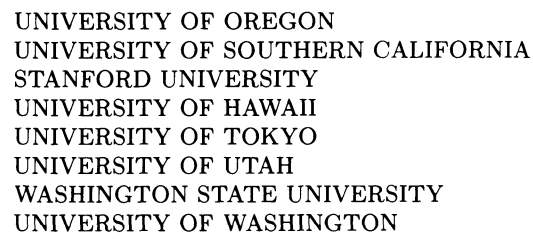

The Supporting Institutions listed above contribute to the cost of publication of this Journal, but they are not owners or publishers and have no responsibility for its content or policies.

Mathematical papers intended for publication in the Pacific Journal of Mathematics should be in typed form or offset-reproduced (not dittoed), double spaced with large margins. Please do not use built up fractions in the text of the manuscript. However, you may use them in the displayed equations. Underline Greek letters in red, German in green, and script in blue. The first paragraph must be capable of being used separately as a synopsis of the entire paper. In particular it should contain no bibliographic references. Please propose a heading for the odd numbered pages of less than 35 characters. Manuscripts, in triplicate, may be sent to any one of the editors. Please classify according to the scheme of Math. Reviews, Index to Vol. 39. Supply name and address of author to whom proofs should be sent. All other communications should be addressed to the managing editor, or Elaine Barth, University of California, Los Angeles, California 90024.

There are page-charges associated with articles appearing in the Pacific Journal of Mathematics. These charges are expected to be paid by the author's University, Government Agency or Company. If the author or authors do not have access to such Institutional support these charges are waived. Single authors will receive 50 free reprints; joint authors will receive a total of 100 free reprints. Additional copies may be obtained at cost in multiples of 50 .

The Pacific Journal of Mathematics is issued monthly as of January 1966. Regular subscription rate: $\$ 190.00$ a year (5 Vols., 10 issues). Special rate: $\$ 95.00$ a year to individual members of supporting institutions.

Subscriptions, orders for numbers issued in the last three calendar years, and changes of address should be sent to Pacific Journal of Mathematics, P.O. Box 969, Carmel Valley, CA 93924, U.S.A. Old back numbers obtainable from Kraus Periodicals Co., Route 100, Millwood, NY 10546.

The Pacific Journal of Mathematics at P.O. Box 969, Carmel Valley, CA 93924 (ISSN 0030-8730) publishes 5 volumes per year. Application to mail at Second-class postage rates is pending at Carmel Valley, California, and additional mailing offices. Postmaster: send address changes to Pacific Journal of Mathematics, P.O. Box 969, Carmel Valley, CA 93924.

PUBLISHED BY PACIFIC JOURNAL OF MATHEMATICS, A NON-PROFIT CORPORATION Copyright (C) 1986 by Pacific Journal of Mathematics 


\section{Pacific Journal of Mathematics}

Vol. 124, No. $2 \quad$ June, 1986

Philip Lee Bowers, Nonshrinkable "cell-like" decompositions of $s \ldots \ldots .257$

Aurelio Carboni and Ross Street, Order ideals in categories .......... 275

Leoni Dalla, Increasing paths on the one-skeleton of a convex compact set in

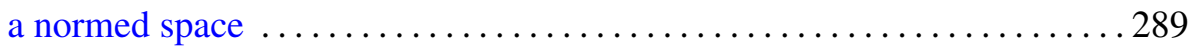

Jim Hoste, A polynomial invariant of knots and links ............... 295

Sheldon Katz, Tangents to a multiple plane curve ................... 321

Thomas George Lucas, Some results on Prüfer rings $\ldots \ldots \ldots \ldots \ldots \ldots 33$

Pham Anh Minh, Modular invariant theory and cohomology algebras of

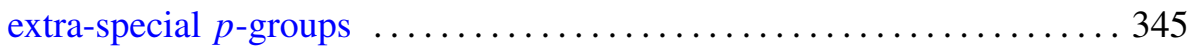

Ikuko Miyamoto, On inclusion relations for absolute Nörlund

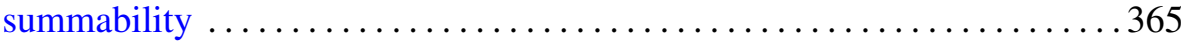

A. Papadopoulos, Geometric intersection functions and Hamiltonian flows on the space of measured foliations on a surface ............. 375

Richard Dean Resco, J. Toby Stafford and Robert Breckenridge

Warfield, Jr., Fully bounded $G$-rings $\ldots \ldots \ldots \ldots \ldots \ldots \ldots \ldots \ldots 4$

Haskell Paul Rosenthal, Functional Hilbertian sums .................417

Luen-Fai Tam, Regularity of capillary surfaces over domains with corners: borderline case ................................. 469

Hugh C. Williams, The spacing of the minima in certain cubic lattices .....483 